\title{
Development of A Novel Gene Expression System for Secretory Production of Heterologous Proteins via the General Secretory (Sec) Pathway in Corynebacterium glutamicum
}

\author{
Huimin Jia ${ }^{1}$, Hedan $\mathrm{Li}^{1}$, Lirong Zhang ${ }^{2}$, Daqing Xu ${ }^{1 *}$ \\ ${ }^{1}$ College of Life Sciences, Agricultural University of Hebei, Baoding 071001, China \\ ${ }^{2}$ Biological Control Centre of Plant Diseases and Pests of Hebei, Agricultural University of Hebei, Baoding 071001, China \\ "Corresponding author: Daqing Xu, College of Life Sciences, Agricultural University of Hebei, Baoding 071000, China. Tel: +86-312-7528251, \\ E-mail: xudaqing@hebau.edu.cn
}

Received: 20 Nov. 2016; $\quad$ Revised: 15 Jan. 2017; $\quad$ Accepted: 17 Mar. 2018; $\quad$ Published online: 18 Apr. 2018

\begin{abstract}
Background: Corynebacterium glutamicum (C. glutamicum) is a potential host for the secretory production of the heterologous proteins. However, to this date few secretion-type gene expression systems in C. glutamicum have been developed, which limit applications of $C$. glutamicum in a secretory production of the heterologous proteins.

Objectives: In this study, a novel and efficient general secretory ( $\mathrm{Sec}$ ) pathway-dependent type gene expression system for the production of heterologous proteins was developed in C. glutamicum.

Materials and Methods: The synthesized cloning/expression cassette $\mathrm{C}$ was assembled into the basic E. coli-C. glutamicum shuttle vector pAU2, generating the Sec-dependent type gene expression vector pAU5. Subsequently, the applicability of the C. glutamicum/pAU5 system was tested using the $\alpha$-amylase AmyE from Bacillus subtilis as a reporter protein.

Results: The vector pAU5 was successfully constructed. The SDS-PAGE experiment showed the AmyE protein band could be observed in the original culture supernatant of the 14067/pAU5-amyE. The Western blotting experiment showed that the AmyE polypeptide could be detected in the culture supernatant of the 14067/pAU5-amyE, not in the cell lysate of 14067/pAU5-amyE. The $\alpha$-amylase specific activity of the culture supernatant of 14067/pAU5-amyE was 103.24 \pm 7.14 U.mg ${ }^{-1}$ protein, while no $\alpha$-amylase activity was detected in the cell homogenate supernatant of 14067/pAU5$a m y E$. These results demonstrate that the recombinant AmyE was efficiently expressed and completely secreted into the extracellular environmentin an active form in C. glutamicum/pAU5 system.

Conclusions: A novel efficient Sec-dependent type gene expression vector pAU5 was constructed in the C. glutamicum. The vector pAU5 employs the strong promoter $t a c-M$ for controlling a constitutive transcription of the target gene, the consensus ribosome binding site (RBS) sequence of $C$. glutamicum to ensure protein translation, and the efficient Sectype cgR_2070 signal sequence to mediate protein secretion in the C.glutamicum. The C. glutamicum/pAU5 system is an efficient expression system for the secretory production of the heterologous proteins.

Keywords: Corynebacterium glutamicum; Gene expression system; General secretory (Sec) pathway; Protein secretion
\end{abstract}

\section{Background}

The secretory production of the recombinant proteins can greatly simplify separation and purification processes and significantly decrease their production costs. Bacillus subtilis is the most widely used host in the industry for the secretory production of the recombinant proteins. However, B. subtilis is mainly applied in the production of its host-derived proteins; in contrast, there exist multiple extracellular and cell wall-associated proteases that degrade heterologous proteins, so attempts to use $B$. subtilis for secretory production of heterologous proteins often has failed (1). Corynebacterium glutamicum has been widely used for the production of amino acids and other low-molecular metabolites (2). Due to its many advantages, such as the GRAS (generally regarded as safe) status (3), the ability to secrete proteins into culture media (4), little extracellular proteolytic activity (5), and the excellent cultural characteristics (6), C. glutamicum has attracted attention as a host for secretory production of the heterologous proteins.

Copyright (C 2017 The Author(s); Published by National Institute of Genetic Engineering and Biotechnology. This is an open access article, distributed under the terms of the Creative Commons Attribution-NonCommercial 4.0 International License (http://creativecommons.org/licenses/ by-nc/4.0/) which permits others to copy and redistribute material just in noncommercial usages, provided the original work is properly cited. 
C. glutamicum possesses two major protein secretory pathways: the general secretory $(\mathrm{Sec})$ pathway that translocates unfolded proteins to the extracellular spacer, and the twin-arginine translocation (Tat) pathway that catalyzes the export of the folded proteins (7). A signal peptide is a short peptide in the $\mathrm{N}$-terminal region of the secretory protein precursor, and it plays a key role in determining secretory pathway and efficiency of the secreted protein. A number of $C$. glutamicum vectors have been developed, some of which were used for the secretory production of heterologous proteins (8-10). Due to the lack of signal sequences in the developed vectors, the two experimental steps were performed to construct secretion-type recombinant vectors. The first step was to fuse the target gene with the signal sequence by an in vitro PCR overlap, followed by the cloning the fused gene into the vector; alternatively, the first step was to clone the signal sequence into the vector, followed by cloning of the target gene downstream of the signal sequence in the vector. The use of secretiontype gene expression vector harboring an efficient signal sequence can simplify the construction process of the recombinant vector and improve the yield of the secreted protein. However, to this date, few secretiontype gene expression vectors in C. glutamicum have been developed $(11,12)$. The lack of secretion-type gene expression vectors limits applications of $C$. glutamicum in the secretory production of the heterologous proteins. Therefore, it is valuable to develop new secretion-type gene expression vectors harboring efficient signal sequences in C. glutamicum. A series of signal peptides have been identified in C. glutamicum (13-19), which lays the foundation for the development of the new efficient secretion-type gene expression vectors.

\section{Objective}

In this study, a novel efficient general secretory (Sec) pathway-dependent type gene expression system for the production of heterologous proteins was developed in C. glutamicum.

\section{Materials and Methods}

\subsection{Strains, Plasmids, and Growth Conditions}

All bacterial strains and plasmids used in this study are listed in Table 1. E. coli was grown in the LB medium at $37 \square(20)$, and C. glutamicum was grown in LBHI medium at $30 \square$ (21). If necessary, kanamycin used was at a concentration of $50 \mu \mathrm{g} . \mathrm{mL}^{-1}$ in the LB medium and $30 \mu \mathrm{g} \cdot \mathrm{mL}^{-1}$ in the LBHI medium.

\subsection{DNA Preparation and PCR Amplification}

Preparation, separation, and purification of the DNA were carried out using the corresponding kits according to the instructions. The synthesis of the primers and the cloning/expression cassette $\mathrm{C}$ and the DNA sequencing were carried out by Sangon (Shanghai, China). The signal-less amyE gene (1878 bp) was amplified using B. subtilis 168 genomic DNA as the template and the forward primer (ATATAGATCTGAAACGGCGAACAAATCGAAT, the underlined bases indicate BglII site) and the reverse primer (ATAT CTCGAGATGGGGAAGAGAACCGCTTAA, the

Table 1. Bacterial strains and plasmids used in this study.

\begin{tabular}{|c|c|c|}
\hline Strain & Description & Reference or Sources \\
\hline E. coli JM109 & 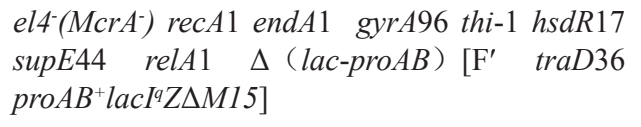 & Stratagene \\
\hline B. subtilis 168 & $\operatorname{trp} C 2$ & (20) \\
\hline C. glutamicum ATCC 14067 & Wild-type $C$. glutamicum & ATCC \\
\hline \multicolumn{3}{|l|}{ Plasmid } \\
\hline pAU2 & $\begin{array}{l}\text { A shuttle vector between } E . \text { coli and } C \text {. } \\
\text { glutamicum, } \mathrm{Km}^{\mathrm{r}}\end{array}$ & $(21)$ \\
\hline pAU5 & $\begin{array}{l}\text { pAU2 (NcoI/Aat II) } \Omega \text { cassette } \mathrm{C}(\text { NcoI/Aat II; } 295 \\
\text { bp, synthesized fragment), A shuttle expression } \\
\text { vector between } E \text {. coli and } C \text {. glutamicum, } \mathrm{Km}^{\mathrm{r}}\end{array}$ & This work \\
\hline pAU5-amyE & $\begin{array}{l}\text { pAU5 }(\text { BglII/XhoI }) \Omega \text { amyE }(B g l \mathrm{II} / X h o \mathrm{I} ; 1878 \\
\mathrm{bp}, B \text {. subtilis } 168 \text { chromosomal DNA }) \text { a } \\
\text { recombinant pAU5 plasmid harboring the amyE } \\
\text { gene }\end{array}$ & This work \\
\hline
\end{tabular}


underlined bases indicate XhoI site) were used for amplification. Annealing temperature and time are $60 \square$ and $15 \mathrm{~s}$, and the PCR reaction was carried out using the high fidelity PrimeSTAR ${ }^{\mathrm{TM}}$ HS DNA polymerase (TaKaRa, Dalian, China) according to the instruction using a Mastercycler (Eppendorf, Hamburg, Germany) PCR machine.

\subsection{Preparation of the Competent Cells and Transformation}

Preparation of the competent $E$. coli and $C$. glutamicum cells as well as DNA transformation was carried out using the methods described by Sambrook et al. and in our previous work $(20,21)$.

\subsection{Construction of the Plasmids pAU5 and pAU5- amyE}

The DNA sequence of the artificially synthesized cloning/expression cassette C (295 bp, flanked by $\mathrm{NcoI}$ and AatII sites) contains the $t a c-M$ promoter, ribosome binding site (RBS) sequence, $c g R \_2070$ signal sequence, multiple cloning sites (MCS) (EcoRI, NotI, BglII, NheI, XhoI, and HindIII), and the His-tag and rrnBT2 terminator (Fig. 1). The cassette $\mathrm{C}$ was digested with $N c o$ I and AatII and then ligated into the E. coli-C. glutamicum shuttle vector pAU2 constructed in our previous study (22), which was also digested with $\mathrm{Nco}$ I and AatII, generating the target plasmid pAU5 (4993 bp) (Fig. 1). The cassette $\mathrm{C}$ in the plasmid pAU5 was sequenced toconfirm its correct insertion. The PCR product of amyE gene was digested with $B g l \mathrm{II}$ and $X h o \mathrm{I}$, and cloned into pAU5, which was also digested with $B g l \mathrm{II}$ and $X h o \mathrm{I}$, generating the recombinant plasmid pAU5-amyE (6865 bp).

\subsection{Flask Cultivation and Sample Preparation of $C$. glutamicum}

Plasmids pAU5 and pAU5-amyE were used to transform C. glutamicum ATCC14067, resulting in ATCC14067/ pAU-5 and ATCC14067/pAU5-amyE, respectively. The seed culture of the $C$. glutamicum strain was prepared by overnight cultivation and was then transferred to 500 $\mathrm{mL}$ flasks containing $100 \mathrm{~mL}$ of the medium for shakeculturing to produce AmyE protein. The cell pellets and culture supernatants of the ATCC14067/pAU-5 and ATCC14067/pAU5-amyE cultures were separated by centrifugation at $13680 \times \mathrm{g}$ for $5 \mathrm{~min}$, respectively. The samples of the culture's supernatants were directly used to test the production of AmyE by SDS-PAGE, Western blotting, and amylase activity analysis. The cell pellets were washed and re-suspended in the phosphate buffer $(\mathrm{pH}$ 6.0) in accordance to 1:20 (v:v) ratio of the buffer:

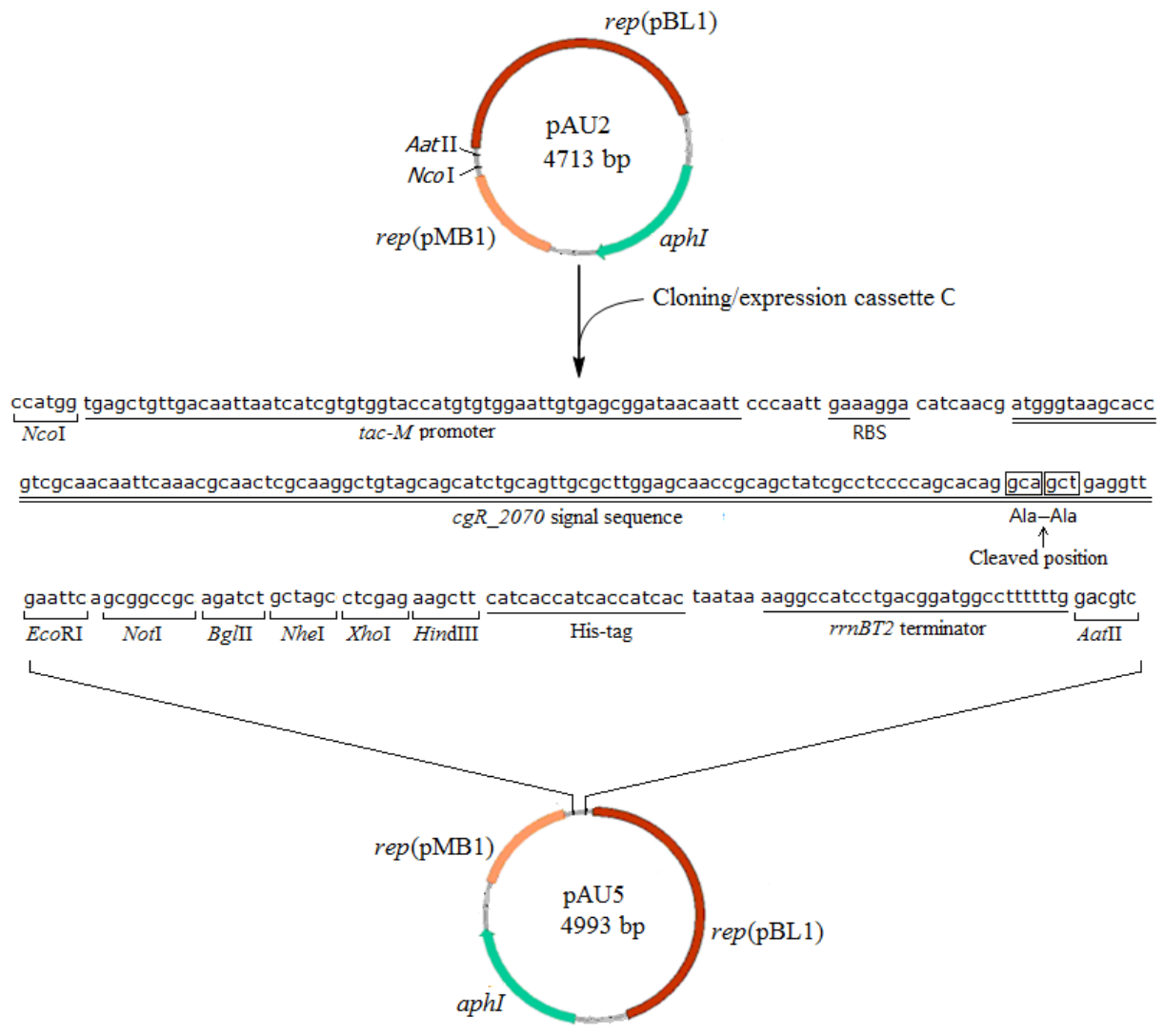

Figure 1. The construction map of the gene expression vector pAU5rep (pMB1), the origin of $E$. coli plasmid pBR322; rep (pBL1), the origin of $C$. glutamicum plasmid $\mathrm{pC} 2$; aphI, kanamycin resistance gene. Nucleotides encoding Ala are boxed, and the peptide bond between the two alanine residues is cleaved position of the signal peptide of CgR2070 by the signal peptidase. 
culture medium. The cell suspension was mixed with a quarter volume of 5X SDS-PAGE loading buffer, boiled for $10 \mathrm{~min}$, and centrifuged in order to remove debris, generating a sample of cell lysate for SDSPAGE and Western blotting. The re-suspended cells were also disrupted by ultrasonication and centrifuged, generating the sample of cell homogenate supernatant for the amylase activity assay.

\subsection{SDS-PAGE and Western Blotting Analysis}

The protein samples $(10 \mu \mathrm{L}$ each) were loaded in the wells of a $12 \%(w / v)$ SDS-polyacrylamide gel. After electrophoresis, the gel was stained using Coomassie Brilliant Blue dye. According to the experimental design, the recombinant AmyE was fused with $6 \times$ Histag at its C-terminus. In order to further confirm production yield and localization, Western blotting was carried out according to the procedure described by An et al. (11). A mouse anti- $6 \times$ His tag antibody (BioDev-Tech, Beijing, China) was used as the first antibody. The second antibody was a goat alkaline phosphatase-labeled anti-His-antibody (BioDev-Tech, Beijing, China), and the polyvinyl difluoride membrane was finally incubated with the color-substrate solution (100 mM Tris, $100 \mathrm{mM} \mathrm{NaCl}, 50 \mathrm{mM} \mathrm{MgCl}, 0.01 \mathrm{mM}$ NBT, $0.01 \mathrm{mM} \mathrm{BCIP,} \mathrm{pH} \mathrm{9.5)} \mathrm{to} \mathrm{observe} \mathrm{color} \mathrm{reaction.}$

\subsection{Analysis of Amylase Activity}

The $\alpha$-amylase activity in the cell homogenate supernatant and the culture supernatant of ATCC14067/ pAU5-amyE were quantitatively analyzed using the standard blue value method (23). Briefly, $20 \mathrm{~mL} \mathrm{2.0 \%}$ $(\mathrm{w} / \mathrm{v})$ of the soluble starch and $5 \mathrm{~mL}$ of phosphate buffer ( $\mathrm{pH}$ 6.0) were mixed and heated at $60 \square$ for 5 min. One $\mathrm{mL}$ of the cell homogenate supernatant or culture supernatant was then added and incubated for $5 \mathrm{~min}$. One $\mathrm{mL}$ of the reaction mixture was added to $5 \mathrm{~mL} \mathrm{I}_{2} / \mathrm{KI}$ solution $\left(\mathrm{I}_{2} 0.088 \mathrm{~g}\right.$. $\mathrm{L}^{-1}$, KI 40 g.L $\left.\mathrm{L}^{-1}\right)$. The absorbance of the reactant was measured at $660 \mathrm{~nm}$. One Unit (U) of the enzyme activity was defined as the amount of amylase required to liquefy $1 \mathrm{~g}$ soluble starch completely for $1 \mathrm{~h}$ at $60 \square$ and $\mathrm{pH}$ 6.0. The determination of the total protein concentration in the both supernatants was carried out using the Protein $\operatorname{dotMETRIC}^{\mathrm{TM}}$ Kit (Sangon, Shanghai, China).

\section{Results}

4.1. Construction of a Novel Gene Expression Vector pAU5 in C. glutamicum

The synthesized cloning/expression cassette $\mathrm{C}$ was assembled into our previously constructed basic $E$.
coli-C. glutamicum shuttle vector pAU2 (22), and DNA sequencing further confirmed its correct assembly, resulting in the target plasmid pAU5 (Fig. 1). The rep (pMB1) of the plasmid pBR322 and the rep (pBL1) of the plasmid $\mathrm{pC} 2$ were used as replication origins of the vector pAU5 in E. coli and C. glutamicum, respectively. The kanamycin resistance gene $a p h I$ was used as the selection marker for both E. coli and C. glutamicum transformants. The cloning/expression cassette of pAU5 employs the MCS (EcoRI, NotI, BglII, NheI, XhoI, and HindIII) to ensure insertion of the target gene, the tac- $M$ promoter and $r r n B T 2$ terminator to control gene transcription, the consensus RBS sequence GAAAGGA of $C$. glutamicumto initiate protein translation, the Sec-type $c g R \_2070$ signal sequence to mediate protein secretion, and the His-tag to simplify purification procedure of the recombinant proteins and provide convenience for their Western blotting analysis in C. glutamicum.

The tac-M promoter was obtained by the site-directed mutagenesis of the tac promoter in our previous work and has been proved to be strong $(22,24,25)$. The $t a c-M$ promoter performs induced transcription in E. coli; in contrast, due to the lack of lacI gene encoding repressor protein on both the vector pAU5 and C. glutamicum chromosome, tac- $M$ performs the constitutive transcription in C. glutamicum. The consensus RBS sequence GAAAGGA ensures an efficient initiation of the protein translation in C. glutamicum (26). The efficient Sec-type cgR_2070 signal sequence was identified by investigating the secretome of the $C$. glutamicum using two-dimensional gel electrophoresis (19). Here, applications of the $t a c-M$ promoter, the consensus RBS sequence of C. glutamicum, and the efficient Sec-type cgR_2070 signal sequence ensure efficient expression and secretion of the recombinant proteins in the C. glutamicum/pAU5 system. Using the C. glutamicum/pAU5 system, the high-efficient secretory production of a recombinant protein was achieved as shown below (Fig. 2).

4.2. Secretory Production of the AmyE in C. glutamicum The exocellular $\alpha$-amylase AmyE from Bacillus subtilis is natively secreted via Sec pathway (27). To test the applicability of pAU5, the signal peptide of AmyE was analyzed using online signal peptide prediction software SignalP 4.1 Server (http://www.cbs.dtu.dk/services/ SignalP), and the signal-less amy $E$ was used as the reporter gene and cloned into pAU5, generating the recombinant plasmid pAU5-amyE. The growth curves showed that growth rate and biomass of ATCC14067/pAU5-amyE were significantly lower than those of ATCC14067/pAU- 
A

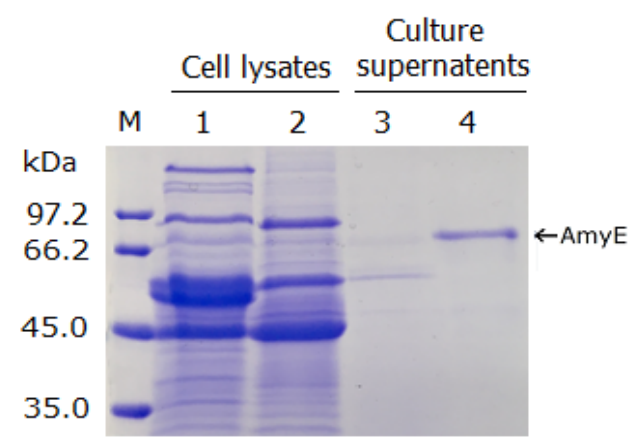

C

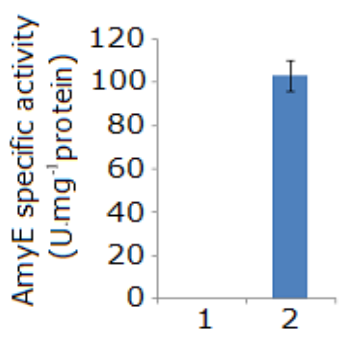

5 (Fig. 3), suggesting that the secretory production of the AmyE protein has negatively affected the cell growth of C. glutamicum.

To determine whether the AmyE sequence was expressed and the protein secreted into the $C$. glutamicum culture medium, the 28-hour-old cultures of 14067/pAU5-amyE and 14067/pAU5 were firstly analyzed by SDS-PAGE. The SDS-PAGE results showed that no specific protein band was observed in the cell lysate of the 14067/pAU5-amyE, while the specific protein band corresponding to the expected molecular mass of AmyE (69 kDa) was observed in the original culture supernatant of 14067/pAU5-amyE (Fig. $2 \mathrm{~A})$. The concentration of the total protein in the culture supernatant of ATCC14067/pAU5-amyE was 0.49 mg.mL ${ }^{-1}$. SDS-PAGE showed that AmyE accounted for the vast majority of the total protein (Fig. 2A, lane 4). Therefore, the concentration of AmyE should be very close to that of the total protein, and it was a good yield for the secretory production of protein. The above results have suggested that the AmyE protein was efficiently expressed and secreted in 14067/pAU5amyE.

To detect whether the expressed AmyE protein was partially retained in C. glutamicum cells, localization of the AmyE polypeptide in the cells and culture supernatant of the 28-hour-old culture of 14067/pAU5$a m y E$ was analyzed by Western blotting using anti-His antibody. As shown in Fig. 2B, the AmyE polypeptide was detected in the original culture supernatant of the
B

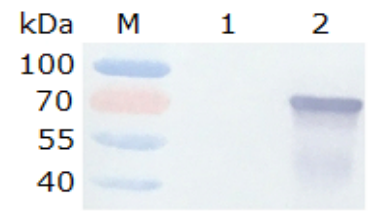

Figure 2. Applicability of pAU5 in C. glutamicum. (A) SDS-PAGE analysis of the culture medium of the different $C$. glutamicum strains. M, the protein molecular weight marker; 1 and 3, 14067/pAU5; 2 and 4, 14067/pAU5-amyE. (B) Western blotting of the AmyE localization in the culture of 14067/pAU5-amyE. M, prestained protein molecular weight marker; 1, cell lysate; 2 , culture supernatant. (C) The specific activity of AmyE in the culture of 14067/pAU5-amyE. 1, cell homogenate supernatant; 2 , culture supernatant. Data expressed as the mean \pm SEM from the three independent experiments.

14067/pAU5-amyE, not in the cell lysate of the 14067/ pAU5-amyE, indicating that the expressed AmyE polypeptide did not accumulate in the $C$. glutamicum cells, and was completely secreted into the culture medium.

The cell homogenate supernatant and the culture supernatant of the 14067/pAU5-amyE were used to measure $\alpha$-amylase activity, respectively. The $\alpha$-amylase specific activity of the culture supernatant

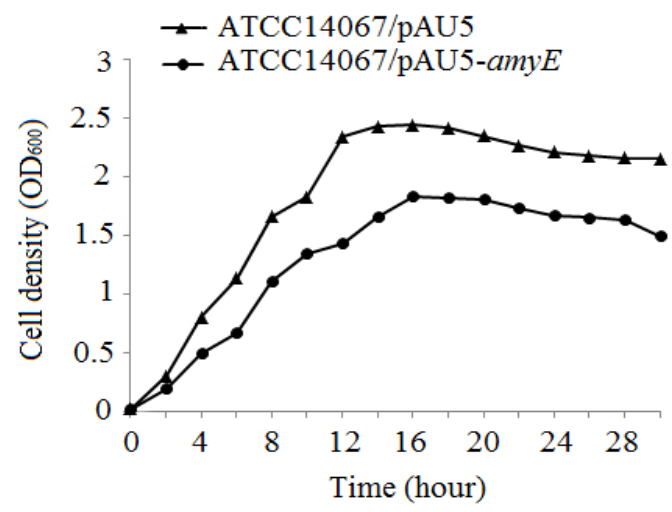

Figure 3. The growth curves of the ATCC14067/pAU5 and ATCC14067/pAU5-amyE strains. According to the same cell concentration at the initial stage of cultivation, the seed cultures of ATCC14067/pAU5 and ATCC14067/ pAU5-amyE were inoculated into $100 \mathrm{~mL}$ LBHI media in $500-\mathrm{mL}$ flasks, respectively. The cultivation was performed at $200 \mathrm{rpm}$ and at $30 \square$, and cell growth was monitored by measuring $\mathrm{OD}_{600}$ of the culture. 
was $103.24 \pm 7.14 \mathrm{U}^{\mathrm{m}} \mathrm{mg}^{-1}$ protein, while no $\alpha$-amylase activity was detected in the cell homogenate supernatant (Fig. 2C) further confirming the efficient expression and secretion of the active-form of AmyE in the 14067/ pAU5-amyE. These experimental results demonstrated high-level of secretory production of the AmyE in $C$. glutamicum/pAU5 system.

\section{Discussion}

Under the condition of flask cultivation, the reporter protein AmyE in the original culture supernatant can be detected by SDS-PAGE analysis, confirming that the $C$. glutamicum/pAU5 system is very efficient. In bacteria, the vast majority of the native extracytosolic proteins are translocated by the Sec pathway (28). Of the identified signal sequences in C. glutamicum, over $90 \%$ are Sec type (18). The high-level expressed AmyE did not accumulate in the cytosol, further confirming the high efficiency of the Sec machinery in C. glutamicum. In comparison to other available vectors, pAU5 possesses the following overall advantages: (i) the vector employs the strong promoter: $\operatorname{tac}-M$, and the consensus RBS sequence of $C$. glutamicum, leading to an efficient expression of the inserted gene; (ii) the vector employs the efficient Sec-type cgR_2070 signal sequence, leading to an efficient secretion of the expressed protein via Sec pathway in C. glutamicum; and (iii) the promoter in pAU5 performs a constitutive expression of the target gene in C. glutamicum, which is more suitable for the large-scale industrial production of the recombinant proteins. These advantages make pAU5 an excellent Sec-dependent type gene expression vector in C. glutamicum.

For secretion of heterologous proteins in $C$. glutamicum, some proteins can be secreted in active forms through either Sec or Tat pathway only (10, 29), while the others can be actively secreted through both Sec and Tat pathways $(18,19)$. Generally, for a certain heterologous protein, there should be a more suitable secretion pathway between $\mathrm{Sec}$ and Tat pathway in C. glutamicum. Therefore, the presence of a different and efficient secretion-type gene expression vectors is valuable in C. glutamicum. Previously, we constructed an efficient Tat-dependent type expression vector pAU3 in C. glutamicum (22). In this study, the efficient Sec-dependent type expression vector pAU5 was constructed. The only difference between pAU5 and pAU3 is the signal sequence. pAU5 and pAU3 constitute a pair of attractive secretion-type sister vectors and would be highly useful for the secretory production of the heterologous proteins in the $C$. glutamicum.

\section{Conclusion}

In this study, a novel and efficient Sec-dependent type gene expression system C. glutamicum/pAU5 was developed. The vector pAU5 employs the strong promoter $t a c-M$ for controlling a constitutive transcription of the target gene, the consensus ribosome binding site (RBS) sequence of C. glutamicum to ensure protein translation, and the efficient Sec-type cgR_2070 signal sequence to mediate protein secretion in $C$. glutamicum. The secretion experiment showed that the reporter protein AmyE was efficiently expressed and secreted using C. glutamicum/pAU5 system. The C. glutamicum/pAU5 system is an efficient expression system for the secretory production of the heterologous proteins.

\section{Acknowledgments}

This work was supported by the National Natural Science Foundation of China (No. 31370141) and the Specialized Research Fund for the Doctoral Program of Higher Education of China (No. 20121302120008).

\section{References}

1. Nguyen TT, Quyen TD, Le HT. Cloning and enhancing production of a detergent- and organic-solvent-resistant nattokinase from Bacillus subtilis VTCC-DVN-12-01 by using an eight-protease-gene-deficient Bacillus subtilis WB800. Microb Cell Fact. 2013;12:79. doi:10.1186/1475-2859-12-79

2. Rohles CM, Gießelmann G, Kohlstedt M, Wittmann C, Becker J. Systems metabolic engineering of Corynebacterium glutamicum for the production of the carbon-5 platform chemicals 5-aminovalerate and glutarate. Microb Cell Fact. 2016;15:154. doi:10.1186/s12934-016-0553-0

3. KumagaiH. Microbial production of amino acids in Japan. In: Scheper T, editor. Advances in biochemical engineering. Berlin: Springer. 2000. Vol.69.p.71-85.

4. Hermann T, Pfefferle W, Baumann C, Busker E, Schaffer S, Bott $\mathrm{M}$, et al. Proteome analysis of Corynebacterium glutamicum. Electrophoresis. 2001;22(9):1712-1723. doi:10.1002/15222683(200105)22:9<1712::AID-ELPS1712>3.0.CO;2-G

5. Yukawa H, Omumasaba CA, Nonaka H, Kos P, Okai N, Suzuki N, et al. Comparative analysis of the Corynebacterium glutamicum group and complete genome sequence of strain R. Microbiology. 2007;153(4):1042-1058. doi:10.1099/ mic. 0.2006/003657-0

6. Yin L, Hu X, Xu D, Ning J, Chen J, Wang X. Co-expression of feedback-resistant threonine dehydratase and acetohydroxy acid synthase increase L-isoleucine production in Corynebacterium glutamicum. Metab Eng. 2012;14(5):542-550. doi:10.1016/j. ymben.2012.06.002

7. Vertes AA. Protein secretion systems of Corynebacterium glutamicum. In: Yukawa H, Inui M, editors. Corynebacterium glutamicum Biology and Biotechnology. Berlin: Springer. 2013;p:351-389.

8. LieblW, Sinskey AJ, Schleifer KH. Expression, secretion, and processing of staphylococcal nuclease by Corynebacterium glutamicum. J Bacteriol.1992;174(6):1854-1861. doi:10.1128/ 
jb.174.6.1854-1861.1992

9. Date M, Yokoyama K, Umezawa Y, Matsui H, Kikuchi Y. Production of native-type Streptoverticillium mobaraense transglutaminase in Corynebacterium glutamicum. Appl Environ Microbiol. 2003;69(5):3011-3014. doi:10.1128/ AEM.69.5.3011-3014.2003

10. Yim SS, An SJ, Choi JW, Ryu AJ, Jeong KJ. High-level secretory production of recombinant single-chain variable fragment $(\mathrm{scFv})$ in Corynebacterium glutamicum. Appl Microbiol Biotechnol. 2014;98(1):273-284. doi:10.1007/s00253-013-5315-x

11. An SJ, Yim SS, Jeong KJ. Development of a secretion system for the production of heterologous proteins in Corynebacterium glutamicum using the Porin B signal peptide. Protein Expres Purif. 2013;89(2):251-257. doi:10.1016/j.pep.2013.04.003

12. Yim SS, Choi JW, Lee RJ, Lee YJ, Lee SH, Kim SY, et al. Development of a new platform for secretory production of recombinant proteins in Corynebacterium glutamicum. Biotechnol Bioeng. 2016;113(1):163-172. doi:10.1002/ bit. 25692

13. Liebl W and Sinskey AJ. Coryneform expression and secretion system. 1990; US patent 4965197.

14. Joliff G, Mathieu L, Hahn V, Bayan N, Duchiron F, Renaud M, et al. Cloning and nucleotide sequence of the cspl gene encoding PS1, one of the two major secreted proteins of Corynebacterium glutamicum: the deduced $\mathrm{N}$-terminal region of PS1 is similar to the Mycobacterium antigen 85 complex. Mol Microbiol.1992;6(16): 2349-2362. doi:10.1111/j.1365-2958.1992.tb01410.x

15. Peyret JL, Bayan N, Joliff G, Gulik-Krzywicki T, Mathieu L, Schechter E, et al. Characterization of the $\operatorname{csp} B$ gene encoding PS2, an ordered surface-layer protein in Corynebacterium glutamicum. Mol Microbiol.1993;9(1):97-109. doi:10.1111/j.1365-2958.1993.tb01672.x

16. Brand S, Niehaus K, Puhler A, Kalinowski J. Identification and functional analysis of six mycolyltransferase genes of Corynebacterium glutamicum ATCC 13032: the genes cop1, $c m t 1$, and $c m t 2$ can replace each other in the synthesis of trehalosedicorynomycolate, a component of the mycolic acid layer of the cell envelope. Arch Microbiol.2003;180(1):33-44. doi:10.1007/s00203-003-0556-1

17. Hartmann M, Barsch A, Niehaus K, Pühler A, Tauch A, Kalinowski J. The glycosylated cell surface protein Rpf2, containing a resuscitation-promoting factor motif, is involved in intercellular communication of Corynebacterium glutamicum. Arch Microbiol. 2004;182(4):299-312. doi:10.1007/s00203004-0713-1

18. 18. Watanabe K, Tsuchida Y, Okibe N, Teramoto H, Suzuki N, Inui $\mathrm{M}$, et al. Scanning the Corynebacterium glutamicum R genome for high-efficiency secretion signal sequences. Microbiology. 2009;155(3):741-750. doi:10.1099/mic.0.024075-0
19. Suzuki N, Watanabe K, Okibe N, Tsuchida Y, Inui M, Yukawa H. Identification of new secreted proteins and secretion of heterologous amylase by Corynebacterium glutamicum. Appl. Microbiol Biotechnol.2009;82(3):491-500. doi:10.1007/ s00253-008-1786-6

20. Sambrook J, Fritsch EF, Maniatis T. Molecular Cloning: $A$ Laboratory Manual. 2nd ed. NowYork: Cold Spring Habor Press; 1989.

21. Xu D, Tan Y, Huan X, Hu X, Wang X. Construction of a novel shuttle vector for use in Brevibacterium flavum, an industrial amino acid producer. J Microbiol Methods. 2010a;80(1):86-92. doi:10.1016/j.mimet.2009.11.003

22. Zhang L, Jia H, Xu D. Construction of a novel twinarginine translocation (Tat)-dependent type expression vector for secretory production of heterologous proteins in Corynebacterium glutamicum. Plasmid. 2015;82:50-55. doi:10.1016/j.plasmid.2015.10.004

23. 23. NSCFFI (National Standardization Center for Food and Fermentation Industry). General metnods of determination for industrial enzymes. In: The food industry standard of People's Republic of China (Fermentation product volume). Beijing: Standards Press of People's Republic of China; 2006;p:117-135.

24. Xu D, Tan Y, Shi F, Wang X. An improved shuttle vector constructed for metabolic engineering research in Corynebacterium glutamicum. Plasmid. 2010b;64(2):85-91. doi: 0.1016/j.plasmid.2010.05.004

25. Xu D, Tan Y, Li Y, Wang X. Construction of a novel promoterprobe vector and its application for screening strong promoter for Brevibacterium flavum metabolic engineering. World $J$ Microbiol Biotechnol. 2011;27(4):961-968. doi:10.1007/ s11274-010-0539-8

26. Amador E, Castro JM, CorreiaA,Martín JF. Structure and organization of the $r r n D$ operon of Brevibacterium lactofermentum: analysis of the 16S rRNA gene. Microbiology. 1999;145:915-924. doi:10.1099/13500872-145-4-915

27. Leloup L, Driessen AJM, Freudl R, Chambert R, Petit-Glatron M. Differential Dependence of Levansucraseand a-Amylase Secretion on SecA (Div) during the Exponential Phase of Growth of Bacillus subtilis. J Bacteriol. 1999;181(6):18201826.

28. de Keyzer J, van der Does C, Driessen AJM. The bacterial translocase: a dynamic protein channel complex. Cell Mol Life Sci. 2003;60(10):2034-2052. doi:10.1007/s00018-003-3006-y

29. Meissner D, Vollstedt A, van Dijl JM, Freudl R. Comparative analysis of twin-arginine (Tat)-dependent protein secretion of a heterologous model protein (GFP) in three different Grampositive bacteria. Appl Microbiol Biotechnol. 2007;76(3):633642. doi:10.1007/s00253-007-0934- 